\title{
Precision of Reinnervation and Synaptic Remodeling Observed in Neuromuscular Junctions of Living Frogs
}

\author{
Stephanie H. Astrow, Vladimir Pitaevski, and Albert A. Herrera \\ Neurobiology Program, Department of Biological Sciences, University of Southern California, Los Angeles, \\ California 90089-2520
}

Repeated in vivo observations were used to study regenerated nerve terminals in neuromuscular junctions of the adult frog Rana pipiens. Sartorius junctions in living animals were stained with the fluorescent vital dye $\mathrm{RH} 414$ and viewed with video fluorescence microscopy. Each junction was observed in the intact muscle and then again 7,10 , and 13 weeks after nerve crush. At 13 weeks, junctions were determined to be mono- or polyneuronally innervated using intracellular recording. Between 7 and 13 weeks, most identified junctions were reinnervated less precisely and completely than described previously. Although some of the original synaptic gutters were reoccupied by regenerated terminal branches, other gutters were only partially occupied, and many appeared abandoned. Junctions showing precise recapitulation of original terminal arborizations comprised a small number of the total examined, as did those where reinnervation was very imprecise. Striking differences in the precision of reinnervation were found within the muscle such that distal terminals regenerated more precisely and completely than did proximal terminals. Terminals in reinnervated muscles were more dynamic than terminals in unoperated muscles over equivalent times. In singly innervated junctions, terminal growth was favored over regression. In doubly innervated junctions, regressive events were more common. Imprecise reinnervation is explained in terms of multisite innervation of muscle fibers and the activity dependence of synaptic stability. We hypothesize that when axons reinnervate the second or third junctions on a fiber, they do so less precisely, because the activity restored by reinnervation of the first junction renders later sites less attractive or less stable.

Key words: reinnervation; synaptic plasticity; synaptic remodeling; neuromuscular junction; frogs; vital dyes; motor endplate; regeneration; in vivo observation; sprouting; motor nerve terminal
When motor nerves are severed and allowed to regenerate into muscle, the number of axons contacting each neuromuscular junction is initially higher than normal. With time, this polyneuronal innervation is reduced by a process similar to developmental synapse elimination, in which motor axons retract some of their connections such that motor unit size and the degree of overlap between motor units are reduced (McArdle, 1975; Rich and Lichtman, 1989). In general, the sartorius muscle of Rana pipiens conforms to this pattern (Werle and Herrera, 1988; Herrera and Werle, 1990). However, $\sim 20 \%$ of sartorius junctions remain polyneuronally innervated for years after reinnervation (Werle and Herrera, 1991). Even in normal intact muscles, polyneuronal innervation is maintained at $\sim 10 \%$ of junctions (Herrera, 1984).

This stability of polyneuronal innervation is somewhat surprising, because frog neuromuscular junctions are known to exhibit substantial plasticity. Unlike similar synapses in mice (Lichtman et al., 1987; Wigston, 1989; Balice-Gordon and Lichtman, 1990; Hill and Robbins, 1991), frog junctions are highly dynamic even in normal intact muscles (Herrera et al., 1990; Chen et al., 1991; Langenfeld-Oster et al., 1993). Cases of apparent synapse elimination and formation of new polyneuronal innervation have been directly observed in frogs (Herrera and Werle, 1990; Herrera et

\footnotetext{
Received Dec. 26, 1995; revised May 30, 1996; accepted June 4, 1996.

This work was supported by National Institutes of Health Grant NS24805 and the Paralyzed Veterans of America, Spinal Cord Research Foundation. We are grateful to Drs. C.-P. Ko, P. B. Sargent, and W. J. Thompson for comments on an earlier version of this manuscript. We also thank $\mathrm{H}$. M. Rupp for technical assistance.

Correspondence should be addressed to Dr. Albert A. Herrera, Department of Biological Sciences, University of Southern California, Los Angeles, CA 90089-2520. Copyright (C) 1996 Society for Neuroscience $0270-6474 / 96 / 165130-11 \$ 05.00 / 0$
}

al., 1990; Herrera et al., 1991). In addition, synapse replacement, wherein the innervation provided by one nerve terminal is substituted by innervation from another terminal, seems to be common in the pectoralis muscle (supracoracoideus) of Xenopus laevis, in which it can be strongly inferred from physiological measurements (Dunia and Herrera, 1993).

One explanation for the apparent stability of polyneuronal innervation in frog muscles may be that reinnervated junctions are truly stable, such that synapse elimination does not occur. True stability implies that synapse elimination can occur only in a certain critical period during development or immediately after reinnervation. Alternatively, the stability could be illusory if there were a dynamic equilibrium between growth and regression of synaptic contacts, such that when a polyneuronal input was eliminated, a new polyneuronal input tended to form at the same or a different junction. We examined these possibilities by repeatedly observing reinnervated neuromuscular junctions in living frogs several weeks after reinnervation was complete. We found that regenerated nerve terminals were more dynamic than intact terminals, suggesting that there is some turnover in polyneuronal innervation. We also found that terminal retraction was more common at doubly reinnervated junctions compared with singly reinnervated junctions. In the latter case, growth was more common. These results suggest that there is ongoing competition at polyneuronally reinnervated junctions. Polyneuronal innervation may persist, because the inputs to such junctions are balanced in their ability to compete. Finally, we carefully compared images of each junction before nerve injury and after reinnervation was complete. We were surprised to find that when junctions were 
given time to regenerate to a stable configuration, most conformed only approximately to their original shape. These observations are discussed in view of a model in which removal of important molecules for specification and maintenance of regenerated synaptic contacts is controlled by activity. A preliminary report has been published (Astrow et al., 1993).

\section{MATERIALS AND METHODS}

Adult Rana pipiens (body length, 59-69 mm; weight, 20-28 gm) were obtained from Lemberger (Oshkosh, WI). Fourteen males and one female were used. Frogs were kept at $24^{\circ} \mathrm{C}$ on a $12: 12 \mathrm{hr}$ light/dark cycle in groups $\leq 12$ before experiments and in individual tanks throughout the in vivo observation period of 13 weeks. They were fed mealworms (Tenebrio molitor larvae) at least twice a week. Neither body length nor weight changed significantly over the observation period. Experiments were performed throughout the year.

In vivo observations. Frogs were anesthetized by immersion in a $0.1 \%$ aqueous solution of tricaine (3-aminobenzoic acid ethyl ester) (Sigma, St. Louis, MO) for $15 \mathrm{~min}$, chilled on ice and subsequently kept at $0-2^{\circ} \mathrm{C}$ throughout the experiment by placing them dorsal side down on a cooled aluminum plate attached to a microscope stage (for details, see Herrera and Banner, 1990). The left hindlimb was supported such that its ventral surface was level. The sartorius was exposed by a skin incision along the ventral thigh and the skin folded back. The fluorescent dye RH414 $(\mathrm{N}$ (3-(triethylammonium)propyl) - 4-(4-( $p$-diethylaminophenyl)butadienyl)pyridinium dibromide) (Molecular Probes, Eugene, OR) at a concentration of $20 \mu \mathrm{M}$ in high $\mathrm{K}$ Ringer's solution containing (in $\mathrm{mM}$ ): $\mathrm{NaCl} 76$, $\mathrm{KCl} 40, \mathrm{CaCl}_{2} 1.8$, TES buffer $5, \mathrm{pH} 7.2$, was superfused onto the left sartorius muscle for $4 \mathrm{~min}$. This dye stains motor nerve terminals by labeling clusters of recycled synaptic vesicle membrane (Betz et al., 1992). The muscle was rinsed with normal frog Ringer's solution containing (in $\mathrm{mm}$ ): $\mathrm{NaCl} 116, \mathrm{KCl} 2, \mathrm{CaCl}_{2} 1.8$, TES buffer 5, $\mathrm{pH} 7.2$, for at least $20 \mathrm{~min}$ and a coverslip was gently placed on the muscle surface. We did not use $\alpha$-bungarotoxin to visualize acetylcholine receptors ( $\mathrm{AChR}$ ) in vivo, because many fibers are innervated by neuromuscular junctions with low safety margins (Grinnell and Herrera, 1980). Inactivity is well known to stimulate junctional remodeling (Wernig and Herrera, 1986). A SIT camera (Dage SIT-66) attached to an Olympus BH2 fluorescence microscope via a $2.5 \times$ photoeyepiece was used to record images on a video cassette recorder. By videotaping images rather than processing them on-line, we were able to conduct observations much faster and thus minimize both the duration of surgery and exposure of the tissue to light. With the dim illumination we used, the resolution of the SIT camera approximated that of the video recorder (240 lines) so that information was not lost.

Images of neuromuscular junctions were made with Plan Neofluar 25/0.80 (Zeiss) and NPL Fl Fluoreszenz 100/1.2 (Leitz) water immersion objectives. The $25 \times$ objective was used briefly $(10 \mathrm{sec})$ to make lowpower images of groups of junctions, which aided their subsequent relocation. High-power images then were taken with the $100 \times$ objective, using vertical illumination from a $100 \mathrm{~W}$ mercury lamp attenuated by means of neutral density filters (0.3-3.1\% transmittance) and an aperture diaphragm. A rhodamine filter set was used (Olympus BP545 and EY475 filters). Individual junctions were illuminated for $\sim 30-40 \mathrm{sec}$. We refer to these conditions as "standard illumination." After the observations, the skin was sutured and sealed with tissue adhesive (Vetseal, Jorgenson Labs, Loveland, CO), and the frog was allowed to recover at room temperature. Recordings were played back through an image processor (Image-1, Universal Imaging, West Chester, PA). The following processing steps were applied to all images shown: (1) averaging 16-64 frames to reduce noise, (2) background subtraction to correct for uneven illumination, (3) mild sharpening to accentuate details using the following convolution masks,

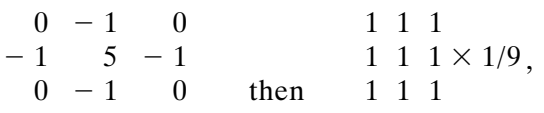

and (4) contrast enhancement. In-focus parts of the junction from different focal planes and overlapping fields of view were pasted together digitally to create composite images. Nerve terminals were traced onto acetate sheets. For purposes of quantifying terminal arbors, lengths of individual branch segments (defined as in Herrera et al., 1990) were measured using a digitizing tablet and Sigmascan (Jandel, San Rafael,
CA). Only neuromuscular junctions on the upper surface of superficial muscle fibers were studied. Between 10 and 40 junctions were observed per muscle.

Immediately after the initial in vivo observation of the left sartorius muscle on day 0 , its nerve was crushed with fine forceps $\sim 2 \mathrm{~mm}$ proximal to the point where it entered the muscle. Thus, the nerve crush was $\sim 2.4$ $\mathrm{mm}$ from the most proximal junctions and $12.3 \mathrm{~mm}$ from the most distal. The right sartorius nerve was similarly crushed after making a small skin incision to expose the site of nerve entry. Four days after the initial observation, the animal was anesthetized as above, and both nerves were crushed a second time in an effort to create higher levels of polyneuronal innervation (Rich and Lichtman, 1989). Postoperative times are described relative to the day of first nerve crush.

Relocation of junctions observed in vivo was straightforward and highly successful. As an aid to relocation, low-power images (2.5/0.08 objective) (Zeiss) of the regions containing observed junctions were made using oblique fiber optic illumination. These images contained blood vessels, nerve trunks, and melanocytes that served as landmarks. In the 15 frogs used, initial observations were made of 471 junctions, and 427 of these $(91 \%)$ were imaged at the second observation time. The success of imaging doubly observed junctions a third time was $97 \%$ (416 of 427), and the success of imaging triply observed junctions a fourth time was $86 \%$ (356 of 416). Most of the attrition was not attributable to a failure to relocate, but to the presence of small, brightly fluorescent cells that sometime overlaid the junction and prevented good imaging of junctional details. For morphometric analysis, statistical significance was determined with either the Mann-Whitney $U$ test or the Wilcoxon signed ranks test.

Electrophysiology. After the final in vivo observation, frogs were killed by decapitation and spinal pithing. The right and left sartorius muscles were excised with nerves dissected centrally to spinal nerves VII and VIII. Nerve-muscle preparations were pinned into SYLGARD-lined Petri dishes filled with Ringer's solution. To determine whether sartorius axons were contained in spinal nerve VII or VIII, or both, nerves were alternately stimulated via suction electrodes and muscle twitches noted. Contractions then were blocked with a minimal concentration of curare (3-4 $\mu \mathrm{M})$. Identified junctions were relocated using the landmarks described above and RH414 fluorescence. For intracellular recording, endplates were viewed through a compound microscope with a water immersion objective (40/0.75) (Zeiss), and muscle fibers were impaled with microelectrodes filled with $3 \mathrm{M} \mathrm{KCl}(10-20 \mathrm{M} \Omega)$. All penetrations were made within $50-100 \mu \mathrm{m}$ of the junction. The presence or absence of polyneuronal innervation was determined by recording endplate potentials (EPPs) in response to $1 \mathrm{~Hz}$ stimulation. Efforts were made to subdivide the EPP by varying the amplitude, polarity, and duration of a stimulus applied to the nerve.

For electrophysiological control experiments, stable intracellular recordings were made from excised preparations for at least $2 \mathrm{hr}$. Resting potentials and miniature endplate potentials (MEPPs) were recorded for varying periods to obtain baselines, then the bathing solution was switched or the junction illuminated as described in Results below. Illumination was done through a Leitz NPL Fl Fluoreszenz 50/1.0 water immersion objective. When using the 40 and $50 \times$ objectives, light intensity was increased to approximate the illumination intensity of the $100 \times$ objective, taking into account the relative magnification and numerical aperture of each objective. Recordings during and after treatment were compared with baseline recordings. Statistical significance was determined with the Mann-Whitney $U$ test.

Histology. Histology was used to verify some of the in vivo observations. After electrophysiological recording, muscles were fixed in the recording dishes and stained with the nitroblue tetrazolium (NBT) method for nerve terminals and with cholinesterase stain (Letinsky and DeCino, 1980). Previous electron microscopic observations showed that NBT precisely reveals the full extent of the nerve terminal (Herrera et al., 1985). Junctions were observed in situ in manually thinned preparations, or short segments of muscle fiber bearing the identified junctions were dissected and mounted individually on slides.

Controls for effects of in vivo observation on synaptic function. Control experiments were necessary to establish that observation with RH414 did not cause or alter the behavior of regenerated terminals. Previously, only mitochondrial dyes have been used for in vivo observation of frog terminals, and these terminals were normal, not regenerated (Herrera et al., 1990; Chen et al., 1991). Exposure to the vehicle solution (high K Ringer's solution) for 4 min produced the expected transient depolarization of muscle fibers, and the addition of $20 \mu \mathrm{M} \mathrm{RH} 414$ did not alter this 

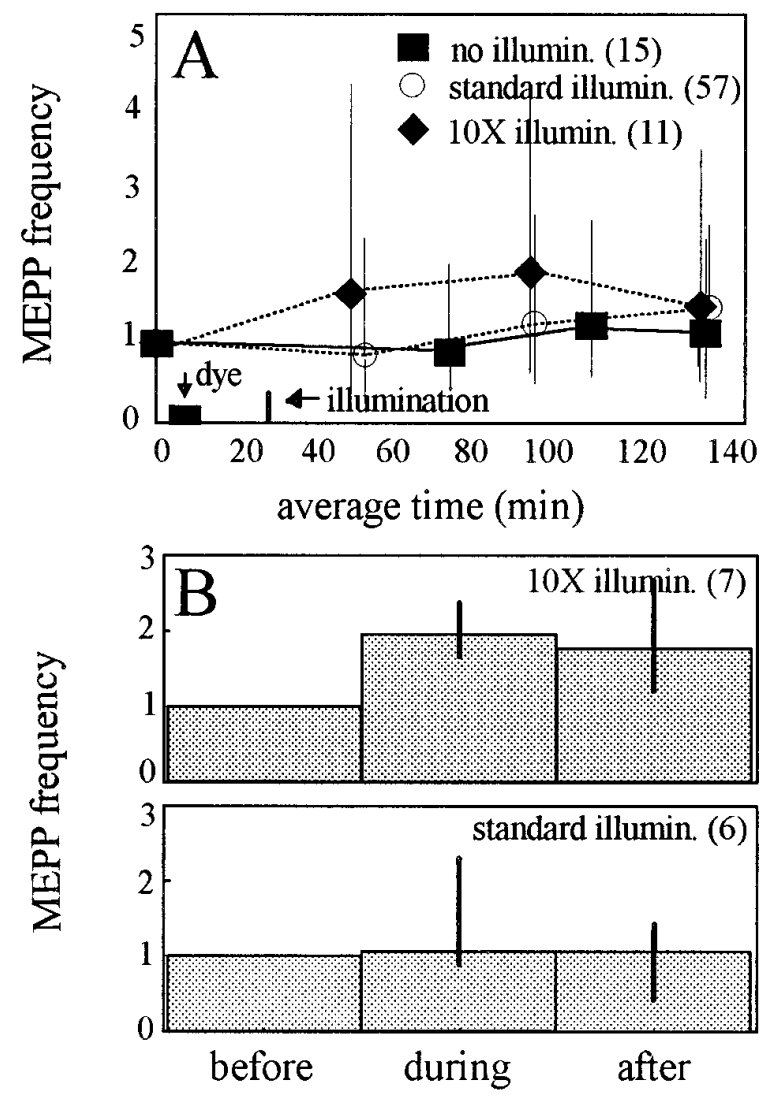

Figure 1. RH414 staining and visualization did not affect synaptic function. $A$ shows the effects of illumination on MEPP frequency in RH414-stained junctions. Approximately $30 \mathrm{~min}$ after applying RH414, junctions received no illumination (solid square), 2 min illumination with the standard intensity used for in vivo observations (open circle), or 2 min illumination with $10 \times$ standard intensity (solid diamond). Standard illumination had no effect on MEPP frequency, whereas $10 \times$ illumination caused a transient increase in MEPP frequency, which persisted for $\sim 2 \mathrm{hr} . B$, Top, Changes in normalized MEPP frequency $30 \mathrm{sec}$ just before, $120 \mathrm{sec}$ during, and $30 \mathrm{sec}$ immediately after illumination with $10 \times$ standard intensity. B, Bottom, Similarly, changes in MEPP frequency $30 \mathrm{sec}$ before, $30 \mathrm{sec}$ during, and $30 \mathrm{sec}$ after illumination with standard illumination. Number of junctions sampled is given in parentheses. Data are medians \pm i.q.r.

effect (data not shown). To test for changes in synaptic function, the frequency of MEPPs was measured before and after staining with RH414. Ringer's solution alone, brief exposure to high K Ringer's solution alone, or high K Ringer's solution with RH414 had no significant long-term effects on MEPP frequency (data not shown).

Having established that dye staining alone did not affect spontaneous quantal transmitter release, we next tested the effects of illumination. Figure $1 A$ shows there were no long-term changes in MEPP frequency when illumination was omitted or when stained junctions were exposed to 2 min of standard illumination (defined above). When the duration of illumination was held constant but intensity increased $10 \times$, there was an $\sim 50 \%$ increase in MEPP frequency, but this returned to normal by $\sim 2 \mathrm{hr}$. To look for transient effects, we recorded MEPPs for a $30 \mathrm{sec}$ period just before illumination, throughout a 30 or $120 \mathrm{sec}$ illumination period, and for $30 \mathrm{sec}$ immediately after illumination (Fig. 1B). Standard illumination for $30 \mathrm{sec}$ had no effect, whereas $10 \times$ standard illumination for $120 \mathrm{sec}$ produced a significant $96 \%$ increase in MEPP frequency. Because the standard procedure did not cause changes in spontaneous quantal transmitter release as measured by MEPP frequency, and was shown to be below the threshold for even transient effects, we concluded that the function of reinnervated neuromuscular junctions was not impaired by in vivo observation.

Controls for effects of in vivo observation on synaptic structure. The absence of short-term physiological changes did not rule out the possibility that in vivo observation caused structural damage that would de- velop more slowly. Therefore, anatomical control experiments also were necessary. Previous studies have shown that the terminals of axotomized motoneurons degenerate over a $2 \mathrm{~d}$ period $(\mathrm{Ko}, 1981)$ and that the earliest regenerating terminals appear at denervated junctional sites $\sim 7$ d after nerve injury (DeCino, 1981; Ding, 1982). We therefore examined regenerated terminals in vivo, then 2-5 d later used the NBT histological method to reexamine the same terminals. We judged that by that time, any damage caused by in vivo observation would be noticeable. In several dozen terminals studied in three muscles, the NBT-stained terminals appeared similar in size and shape to their appearance several days earlier. In addition, the histological appearance of observed junctions was compared with unobserved junctions in the same muscle as well as with junctions in the contralateral unobserved muscle. No differences were detected in general appearance, incidence of sprouting or retraction, total nerve terminal length, number of nerve terminal branches, or number of axonal inputs. We concluded that the structure of reinnervated neuromuscular junctions was not altered by in vivo observation.

\section{RESULTS \\ RH414 reliably stained regenerated nerve terminals}

RH414 should be a reliable vital stain for regenerated nerve terminals (Barry and Ribchester, 1995), because staining depends on transmitter release (Betz et al., 1992) and regenerated terminals soon recover nearly normal levels of release (DeCino, 1981; Ding, 1982). To verify this expectation, sartorius nerves were crushed, then 1-13 weeks later, reinnervated muscles were stained in vivo by irrigating for 4 min with $20 \mu \mathrm{M}$ RH414 in high K Ringer's solution. After rinsing with normal Ringer's solution, the anesthetized frogs were killed and muscles immediately excised and stained with the NBT method. We found that RH414 stained the full extent of the regenerated nerve terminals revealed by NBT. In addition, RH414 stained preterminal axons, "escaped" axons extending beyond the tips of synaptic gutters, terminal sprouts, sensory terminals within muscle spindles, and autonomic axons associated with blood vessels. Stained non-neuronal elements included Schwann cells, myelin sheaths, and interstitial cells scattered in the superficial connective tissue. In general, the staining patterns of neuronal and non-neuronal cells we observed in vivo strongly confirmed the in vitro observations of Betz and colleagues (Betz et al., 1992).

It was important to determine whether non-neuronal cells, in particular the processes of Schwann cells, could be mistaken for nerve terminals after RH414 staining. To test this possibility, the sartorius nerve in one leg was severed and deflected in such a way that reinnervation of the sartorius muscle would not occur for $\sim 4$ weeks. Approximately 2 weeks after the operation, the denervated sartorius and the contralateral innervated sartorius were dissected and stained together with RH414 and rhodamine conjugated $\alpha$-bungarotoxin. RH414 did not stain denervated junctions, identified by their $\alpha$-bungarotoxin staining, although muscle fibers in the denervated muscle did show an increase in background staining compared with fibers in reinnervated or intact muscles. Surprisingly, few RH414-stained Schwann cell bodies were seen at denervated junctions (data not shown), although Schwann cell bodies often were well labeled in innervated junctions (Figs. 2, 3). We concluded that the Schwann cell staining frequently observed in intact and reinnervated RH414-stained muscles was principally associated with the cell body and thus could be distinguished from the labeling of motor nerve terminals.

\section{Reinnervation of neuromuscular junctions was neither precise nor complete}

Repeated in vivo observation revealed the junctions were reinnervated with a surprising degree of imprecision and incompleteness and that regenerated terminals were highly dynamic. The photo- 

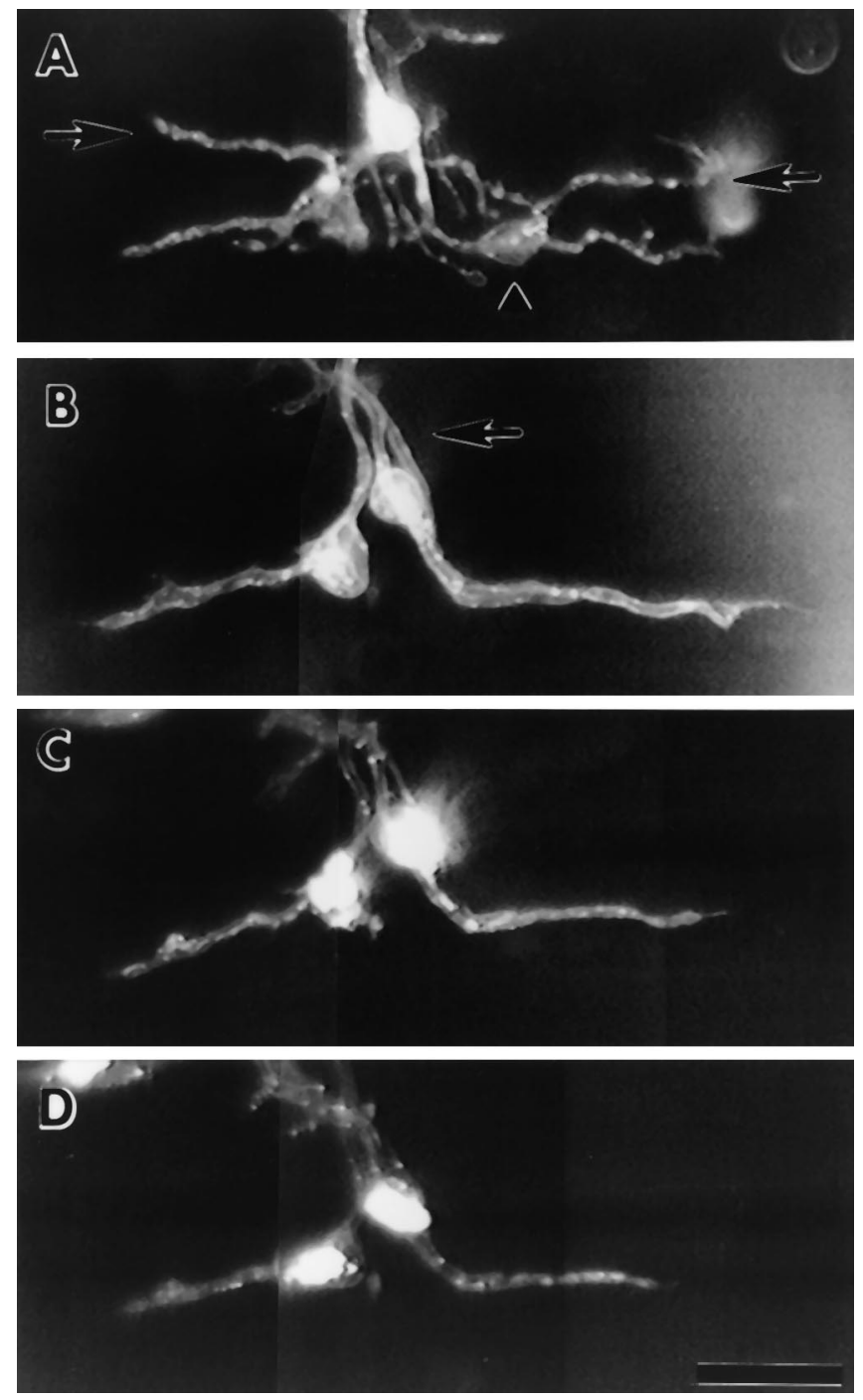

Figure 2. Some original synaptic gutters were reoccupied completely, whereas other gutters were reoccupied incompletely or abandoned altogether. This example shows such a junction on the day of the first nerve crush when terminals were intact $(A)$, then 7 weeks later $(B), 10$ weeks later $(C)$, and 13 weeks later $(D)$. Abandoned original branches are indicated by arrows in $A$. Arrow in $B$ indicates three axonal inputs. Intracellular recording showed that the junction was doubly innervated. The bright oval objects are Schwann cell somata, one of which (arrowhead in $A$ ) disappeared between 0 and 7 weeks. Scale bar, $30 \mu \mathrm{m}$.

graphs in Figures 2-5 illustrate the range of our observations, and results are summarized in the graphs in Figures 6-10. Figures 2-5 show observations on the day of the first nerve crush when terminals were intact $(A), 7$ weeks later $(B), 10$ weeks later $(C)$, and 13 weeks later $(D)$. Figure 2 illustrates the most common result. Figure $2 A$ shows the original appearance of the intact RH414-stained nerve terminal. Control observations (data not shown) revealed a nearly perfect correspondence between the terminal and underlying AChRs at most junctions. Thus, it could be safely assumed that the intact terminal was a faithful indicator of the original synaptic sites. Comparison of Figure 2, $A$ and $B$, shows that the two lower branches of the junction were reinnervated fairly precisely at 7 weeks. The two upper branches (arrows in Fig. 2A) and several other small branches of the original junction were not reinnervated at 7 weeks. Although it is possible that these sites were transiently reinnervated before 7 weeks, these uninnervated sites appeared to be abandoned, because they were still not reoccupied at 10 weeks (Fig. 2C) or 13 weeks (Fig. $2 D)$. The need to perform intracellular recording precluded the use of $\alpha$-bungarotoxin to visualize AChRs after the final observation. It is presumed at that the nonreinnervated sites lost AChRs (Krause and Wernig, 1985; Grinnell and Do, 1991; Werle and Sojka, 1996), but this was not directly visualized. Intracellular recording at 13 weeks revealed that the junction shown in Figure 2 was doubly innervated. Indeed, at least two axonal inputs can be seen in each view of the reinnervated junction (arrow in Fig. 2B), although it is not clear which input corresponded to which step in the two-step EPP. As was commonly seen for terminals of many doubly innervated junctions, the branches of these terminals progressively retracted between 7 and 13 weeks. Both branches shortened by approximately the same amount, with no evidence for selective retraction or growth of one input over the other. There also were changes in Schwann cell perikarya, with one of the three Schwann cells disappearing between 0 and 7 weeks (arrowhead in Fig. 2A). Whether this denervated Schwann cell migrated away or degenerated could not be determined.

A similar example that illustrates other common features is shown in Figure 3. Recordings revealed that this junction also was doubly innervated. Comparison of Figure 3, $A$ and $B$, shows that in the left half of the junction, nearly all of the former gutters were reoccupied. However, neither terminal branch on the left was confined to the old sites. The lower branch extended $\sim 25 \mu \mathrm{m}$ beyond its former distal tip. Histological staining at 13 weeks confirmed that this extended region was a synaptic contact. There was also a new axon making contact with the lower branch (arrowhead in Fig. 3B), but whether this was an input or output could not be determined. The terminal in the upper branch also grew beyond its former distal tip, leaving the fiber as a sprout (arrow in Fig. 3B) that could be traced for $150 \mu \mathrm{m}$ before terminating in a synaptic contact on the adjacent fiber (data not shown). The upper branch developed two new inputs or outputs, one between 7 and 10 weeks (arrowhead in Fig. 3C) and one between 10 and 13 weeks (arrowhead in Fig. 3D). In contrast to the left half of the junction, the right half was reoccupied incompletely. By 7 weeks (Fig. 3B), the upper branch was only $34 \%$ of its former length of $102 \mu \mathrm{m}$. This part of the terminal slowly lengthened to $39 \%$ of its former length at 10 weeks (Fig. 3C) and 56\% at 13 weeks (Fig. 3D), although it remained unbranched. The short lower branch near the center of the original junction was not reoccupied during the observation period. In many junctions such as this, the terminal formed stable synaptic contacts on patches of sarcolemma that were not previously seen to be innervated (distal tip of lower branch on the left) while abandoning former synaptic gutters in the vicinity (lower branch on the right). The three terminal Schwann cells persisted throughout the 13 weeks, but the somata of the two on the left $(1,2$ in Fig. $3 A)$ moved to the left by $\sim 30 \mu \mathrm{m}$ between 0 and 7 weeks.

In a few cases, regeneration was so faithful to the original pattern that the appearance of the former terminal was completely recapitulated. It seemed that such cases were more common in junctions most distant from the point of nerve entry, an observation that was confirmed quantitatively (see below). In the example in Figure 4, the 7 week regenerated terminal (Fig. 4B) was nearly congruent to the original intact terminal (Fig. $4 A$ ). The regenerated terminal remained unchanged at 10 weeks (Fig. $4 C$ ) and 13 weeks (Fig. 4D). It is highly unlikely that these results were attributable to an inadvertent failure to denervate this junction. In 

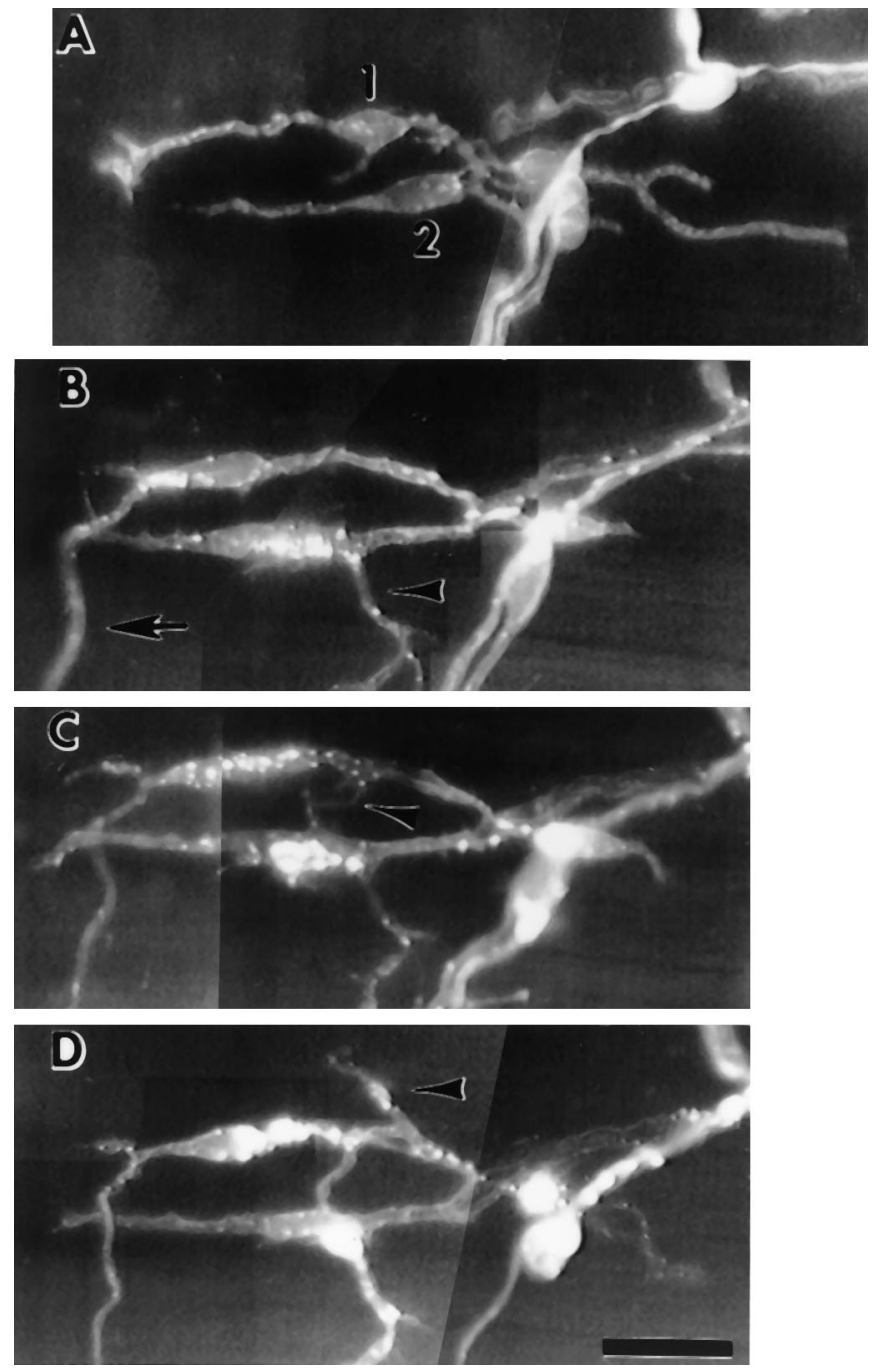

Figure 3. Former gutters can be reoccupied slowly. In this example, gutters on the left were nearly completely reoccupied, whereas reoccupation of gutters on the right was initially incomplete but progressed slowly. Observation times as in Figure 2. Both branches on the left grew beyond their former distal tips. The upper branch on the left produced an "escaped" axon (arrow) that formed a synapse on the fiber below (data not shown). The lower branch on the left formed an extended synaptic contact. Three new axonal inputs or outputs appeared (arrowheads). The Schwann cell somata numbered 1 and 2 moved to the left between 0 weeks $(A)$ and 7 weeks $(B)$. Scale bar, $30 \mu \mathrm{m}$.

our hands, even a single nerve crush is $100 \%$ effective in achieving complete denervation (Werle and Herrera, 1988). Nerves in the present preparations were twice crushed. In addition, the preterminal axon arose from an intramuscular nerve bundle that contained refractile inclusions typical of reinnervated nerve sheaths.

At the other extreme, there were occasional cases of regenerated terminals that bore so little resemblance to their former appearance that they could barely be recognized. In such cases, identification was verified by noting small distinguishing characteristics and by the unique position of the junction relative to distinctive junctions, nerves, and blood vessels in the immediate surround. Figure 5 shows two adjacent junctions that were reinnervated very imprecisely. For both junctions, only vague similarities could be appreciated between the 7 week regenerated terminals (Fig. $5 B$ ) and the original intact terminals (Fig. $5 A$ ). Some of the original synaptic gutters appeared to be reinnervated, but
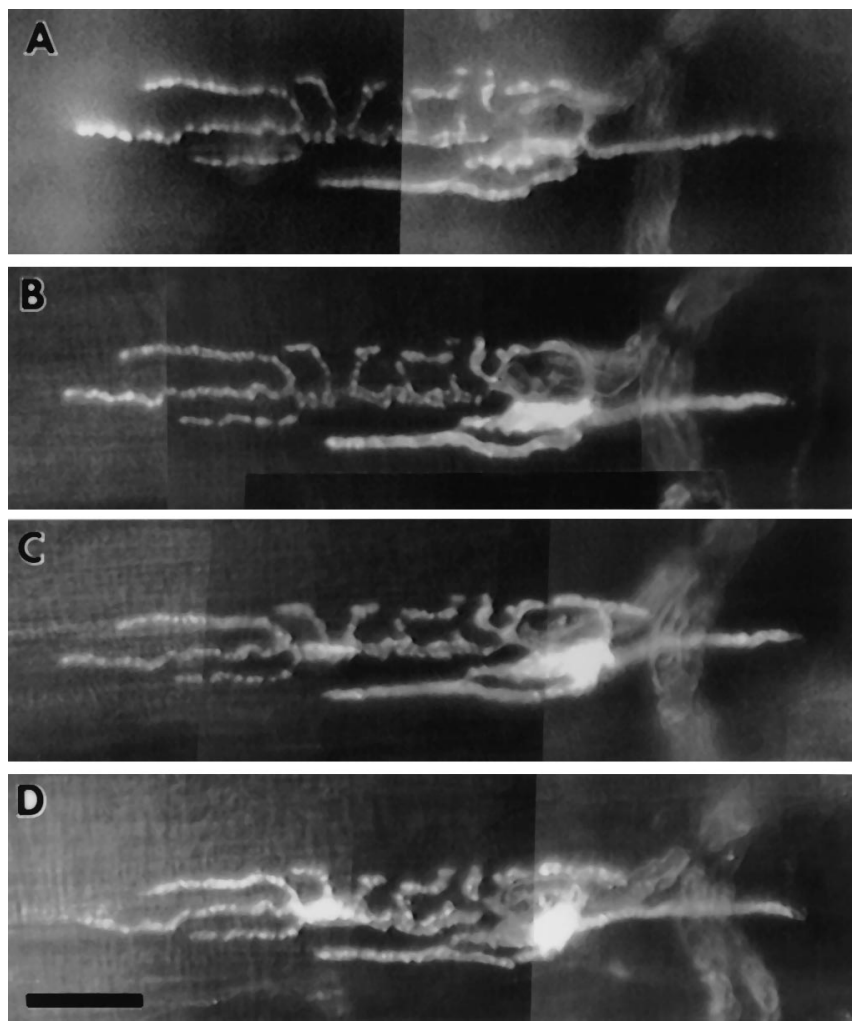

Figure 4. In a minority of cases, regeneration faithfully recapitulated the original appearance of the nerve terminal. Observation times as in Figure 2. After regeneration, there was no appreciable growth or remodeling. Scale bar, $30 \mu \mathrm{m}$.

the branching pattern of the nerve was quite different. The upper junction showed little remodeling between 7 weeks (Fig. 5B), 10 weeks (Fig. 5C), and 13 weeks (Fig. 5D), although there was some redistribution of bright spots. Between 7 and 13 weeks, the lower junction showed substantial regression of a twofold nature. First, there was a reduction in the extent of the terminal and the number of branches. Second, the number and size of RH414-labeled spots was sharply reduced. Because EPP size has been shown to be proportional to the number of vesicle clusters stained with a similar dye (Betz et al., 1993), it is possible that synaptic efficacy of the lower junction was similarly reduced.

Quantitative analyses verified that reinnervation of neuromuscular junctions tended to be imprecise and incomplete at long postoperative times. To quantify the precision of reinnervation, tracings of the last view of the regenerated terminal (usually at 13 weeks) were superimposed onto the view of the intact terminal. The fraction of the 13 week terminal located at sites occupied by the 0 week terminal was measured. This fraction ranged from near 0 in cases in which little of the regenerated terminal lay in synaptic gutters occupied by the intact terminal (e.g., Fig. 5), to near 1 when most of the regenerated terminal was located in previously occupied gutters (e.g., Fig. 4). Liberal criteria for matching were defined and consistently applied so that differences attributable to mechanical distortion or altered angles of viewing were not scored as mismatches. Results for 112 representative junctions are shown in Figure $6 A$. Values for the index of precise reinnervation varied widely, ranging from 0.17 to 0.98 with a mean value of 0.57 (arrow in Fig. $6 A$ ). If accurate reinnervation is arbitrarily defined as cases in which $>80 \%$ of the regenerated terminal was located in former synaptic sites, then only $13 \%$ of the junctions (15 of 112) fell into 

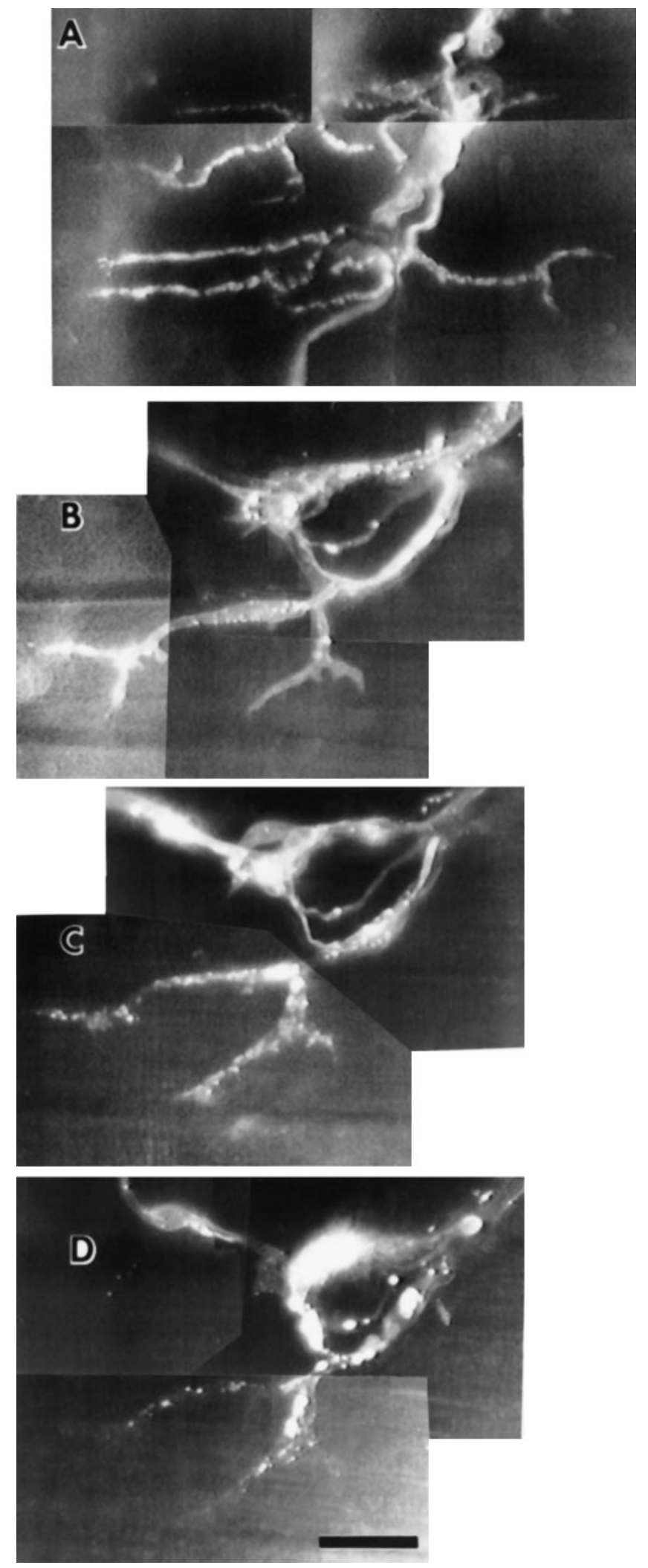

Figure 5. Occasionally, reinnervation was so imprecise and incomplete that the junction was barely recognizable. $A$ shows the original appearance of two junctions on adjacent fibers. Observation times as in Figure 2. After regeneration, the configuration upper terminal was fairly static, but there was rearrangement of RH414-stained vesicle clusters. The lower terminal showed substantial regression in overall size and in the size and number of vesicle clusters. Scale bar, $30 \mu \mathrm{m}$.
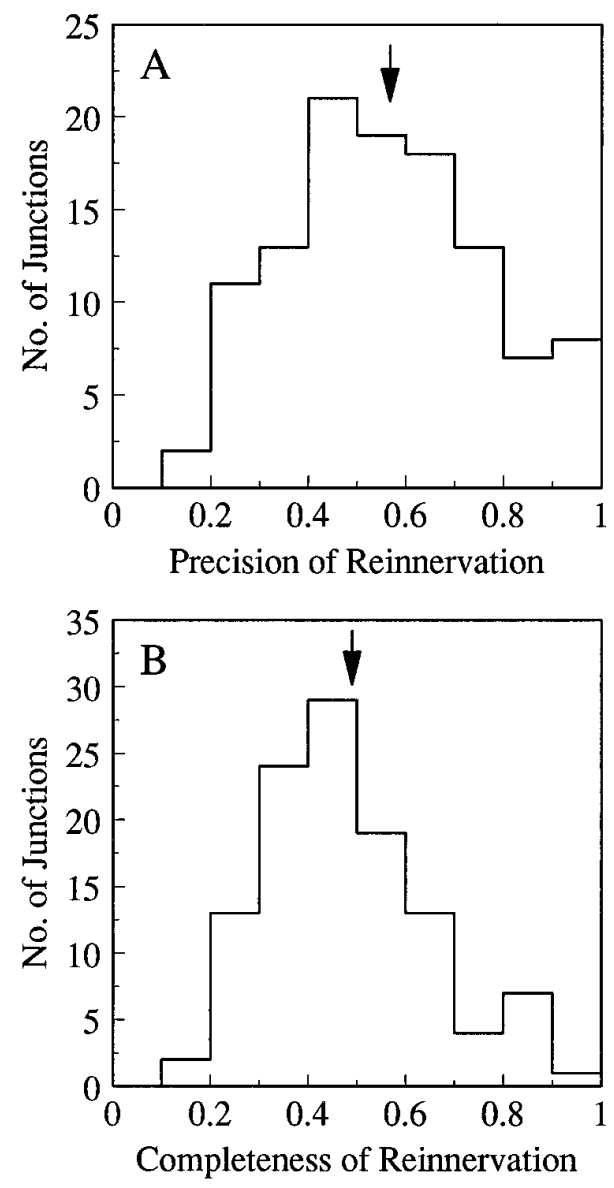

Figure 6. Reoccupation of former synaptic sites tended to be imprecise and incomplete at long postoperative times. Graphs show frequency distributions of indices for precision of reinnervation $(A)$ and completeness of reinnervation $(B)$ for a representative set of 112 junctions. The indices are defined in Results. Arrows indicate mean values of 0.57 in $A$ and 0.49 in $B$.

this category. If inaccurate reinnervation is defined as cases in which half or less of the regenerated terminal occupied former sites, then $42 \%$ of junctions (47 of 112) fell into this category. Imprecise, inaccurate reinnervation was clearly the more common result.

Similar results were obtained for measures of the completeness of reinnervation at the end of the observation period. To quantify the completeness of reinnervation, tracings of the 0 and 13 week terminals again were superimposed. The fraction of the original synaptic sites occupied by regenerated nerve terminals at 13 weeks was determined. This fraction ranged from near 0 when little of the original gutter was reoccupied, to near 1 when the original gutter was completely reoccupied. Results are shown in Figure $6 B$. Again, values varied widely, from 0.18 to 0.98 . Reinnervation tended to be fairly incomplete, with an average value of 0.49 (arrow in Fig. 6B). In $60 \%$ of the junctions (68 of 112), only half or less of the original gutter was reinnervated at 13 weeks. Nearly complete reinnervation of former sites was a rare result, with only $7 \%$ of junctions ( 8 of 112) having indices greater than 0.8 .

\section{Nerve terminal growth differed in singly and doubly reinnervated neuromuscular junctions}

Despite the fact that former synaptic sites were reoccupied imprecisely and incompletely, nerve terminals tended to grow to approximately their former lengths. To summarize the time 


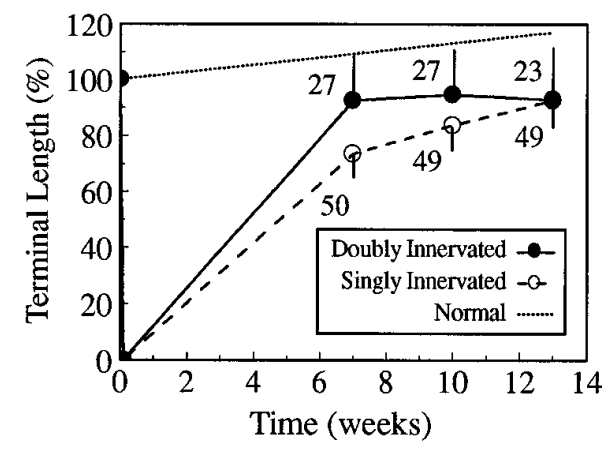

Figure 7. Nerve terminals in singly and doubly innervated junctions regenerated at different rates. The graph shows the time course of changes in total nerve terminal length. Data are expressed as a percentage of the value at time 0 , which is the day the nerve was crushed the first time. Points are mean \pm SEM for identified singly innervated (open circle) and polyneuronally innervated (solid circle) neuromuscular junctions. Number of junctions analyzed at each time point is indicated. The dotted line depicts growth predicted by interpolation from studies of normal intact junctions (Herrera et al., 1990).

course of regeneration, the length of all terminal branches was summed at each observation and expressed as a percentage of the length of the original intact terminal. To test whether the restoration of terminal length was correlated with the number of reinnervating motoneurons, intracellular recording was used to determine whether junctions were singly or multiply innervated (see Materials and Methods). Although it is possible that axonal branches from one, two, or more motoneurons can reinnervate a muscle fiber at a given neuromuscular junction, all junctions in our study were found to be either singly or doubly innervated. Results for 50 singly innervated junctions and 27 doubly innervated junctions are presented. Figure 7 shows that the initial growth of terminals (measured at 7 weeks) was significantly slower in singly innervated junctions than doubly innervated junctions $(p<0.05)$. Between 7 and 13 weeks, singly innervated junctions continued to grow steadily, however, whereas doubly innervated junctions showed no change. In fact, the growth of singly innervated junctions was greater than would be expected for normal intact terminals over a 13 week period (data from Herrera et al., 1990) (compare slopes of the dashed and dotted lines in Fig. 7). Differences in nerve terminal growth at singly and doubly innervated junctions between 7 and 13 weeks could be seen more clearly by examining the distribution of terminal length changes, as in Figure 8 . The arrows in Figure 8 indicate that the mean change was $26 \%$ growth in singly innervated junctions, more than six times higher than the $4 \%$ growth seen in doubly innervated junctions. This difference was highly significant $(p<0.01)$.

The finding that regeneration tended to restore the full terminal length (Fig. 7), but did so imprecisely (Fig. 6), provided quantitative support for a conclusion that was initially drawn from visual inspection of the fluorescence images. This conclusion was that during regeneration, synaptic contacts were frequently formed at sites that were not previously seen to be innervated. Unoccupied former sites in the vicinity appeared to be less attractive than these new sites.

\section{Remodeling involved disproportionate changes within neuromuscular junctions}

The remodeling seen in reinnervated junctions was not characterized by simple, proportionate growth or shrinkage of nerve terminals. Within single junctions, it was common for some segments
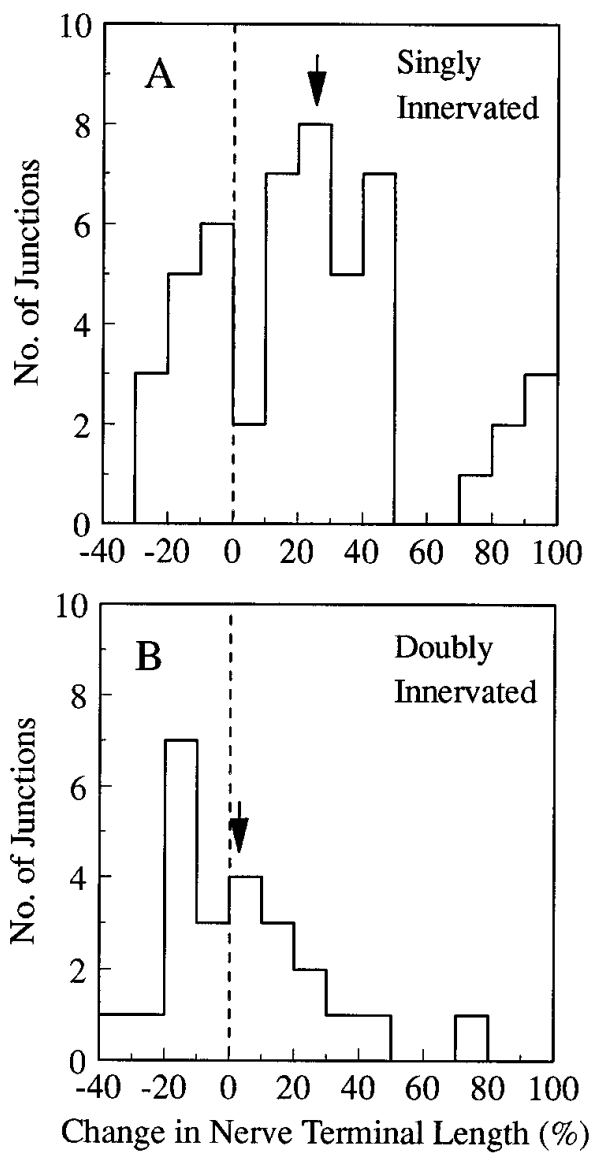

Figure 8. Once regeneration was complete, terminals at singly reinnervated junctions grew more than 6 times faster than terminals at doubly reinnervated junctions. The graphs show changes in total nerve terminal length between 7 and 13 weeks after double nerve crush, for 49 singly innervated $(A)$ and 24 doubly innervated $(B)$ junctions. Changes in length are expressed as a percentage of the terminal length at 7 weeks. Arrowheads indicate means of $26 \%$ in $A$ and $4 \%$ in $B$. The distributions are significantly different $(p<0.01)$. In $A$, the $91-100 \%$ bin includes two values greater than $100 \%(135 \%, 174 \%)$.

of the terminal to grow substantially or to retract between 7 and 13 weeks, whereas other branches appeared and disappeared. At the same time, total terminal length could increase, decrease, or show no change. Figure $9 A$ illustrates how such changes were analyzed. Between the initial view (upper drawing) and final view (lower drawing) in this schematic but typical example, the number of branch segments (numbered) decreased from seven to five. Branch segments were defined as portions of the terminal between two branch points or between a branch point and a distal tip. The number of branch points (lettered) also decreased, from three to two. These changes were attributable to the disappearance of the branch segment numbered 4 in the initial view. Thus, it was considered that two branch segments did not change (initial branches 1 and 6), two segments grew (initial branches $3+5$ and 7 ), and two segments retracted (initial branches 2 and 4). Note that the disappearance of branch segment 4 made it impossible to identify former branch point $\mathrm{C}$. Despite the remodeling and redistribution shown in this example, total nerve terminal length did not change.

The nonuniformity of remodeling within individual junctions is analyzed quantitatively in Figure $9, B$ and $C$, for 47 singly inner- 

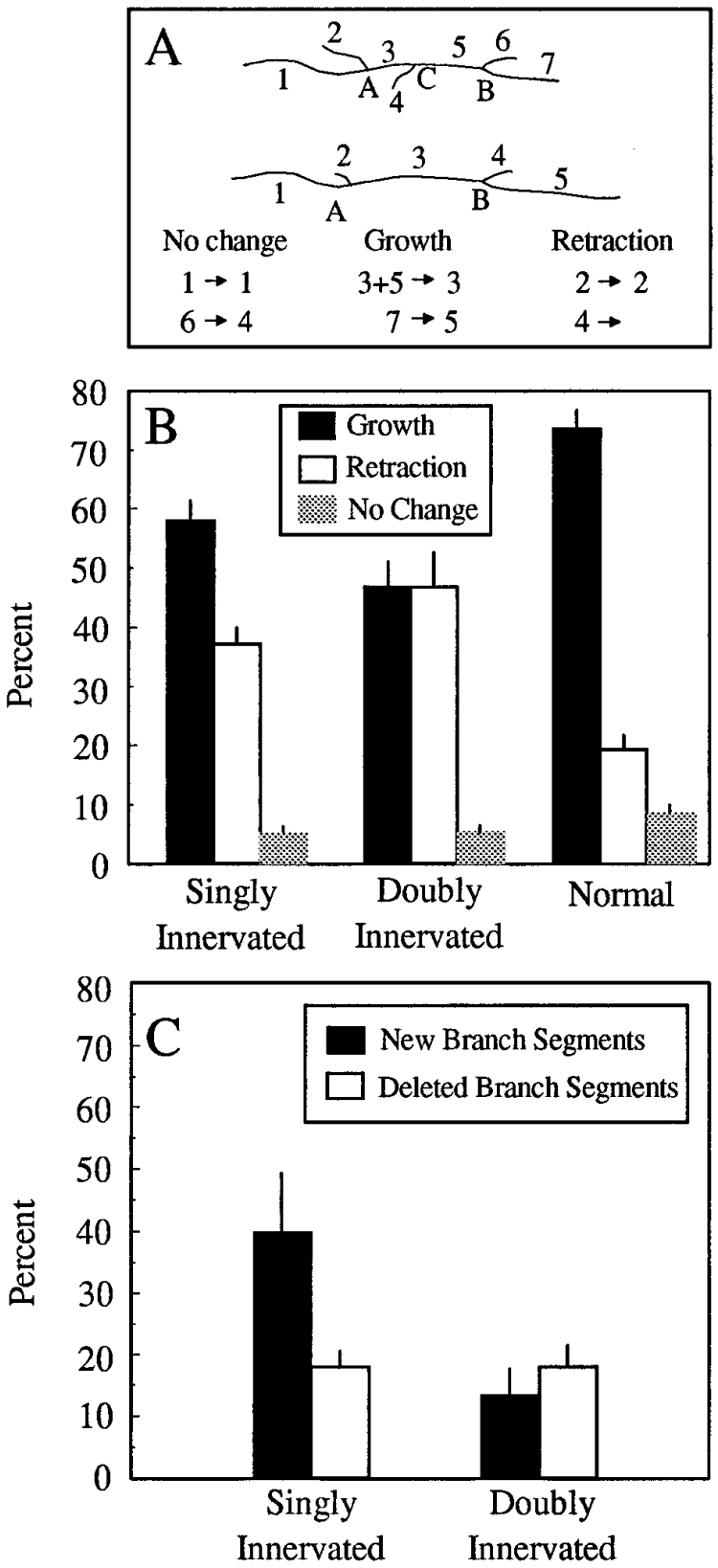

Figure 9. Within individual junctions, different parts of the terminal arborization could grow, retract, or remain unchanged, and this differed with single and double reinnervation. $A$ shows the typical appearance of a regenerated nerve terminal at 7 weeks (upper drawing) and 13 weeks (lower drawing). Terminal branch segments are numbered, and branch points are lettered as described in Results. The legend at the bottom of $A$ indicates which branch segments showed no change, growth, or retraction. In $B$, the left and center triplets of bars describe the fate of regenerated terminal branches that were present at both the 7 week and 13 week observations in 47 singly innervated junctions (left) and 23 doubly innervated junctions (center). The right triplet of bars in $B$ shows results from 77 normal intact terminals observed at an interval of 18 weeks (data from Herrera et al., 1990). The height of the bars represents the mean percentage of branch segments that grew (black), retracted (white), and did not change (gray). $C$ shows the occurrence of new branch segments or branch segments that were deleted between 7 and 13 weeks, for singly and doubly innervated junctions. The number of new and deleted branch segments at 13 weeks is expressed as a percentage of the number of branch segments at 7 weeks. vated junctions, 23 doubly innervated junctions, and 77 normal intact terminals. The normal terminals were observed in an earlier study (Herrera et al., 1990) over a longer interval (18 weeks), but results shown here are normalized to a 6 week interval to allow direct comparison to results from reinnervated junctions. Figure $9 B$ describes the fate of branch segments that were present at both 7 and 13 weeks. For singly innervated junctions, a majority $(58 \%)$ of the segments grew as expected, because there was $26 \%$ growth in total terminal length (Fig. 8). However, a substantial fraction $(37 \%)$ of the segments of these terminals actually retracted. For doubly innervated junctions, nearly half $(47 \%)$ of the segments retracted, whereas an equal percentage $(47 \%)$ grew. The percentage of growing branches was significantly higher in singly innervated junctions, and the percentage of retracting branches was significantly higher in doubly innervated junctions $(p<0.05)$. Normal intact terminals showed a significantly higher percentage (74\%) of growing branch segments than either singly or doubly reinnervated terminals $(p<0.01)$. There was concurrent retraction in $19 \%$ of the intact terminals, significantly less than either singly or doubly reinnervated terminals $(p<0.01)$. Growth in overall length was $18 \%$ for the intact terminals.

A comparison of the patterns of growth seen in terminals at singly reinnervated and normal junctions is illuminating. Terminals at singly reinnervated junctions grew more than intact terminals (26 vs $18 \%$, respectively). Regenerated terminals achieved this growth in one third the time required by intact terminals (6 vs 18 weeks). Although regenerated terminals grew more in total size, however, fewer of their branch segments lengthened (58 vs $74 \%$ ) and more retracted (37 vs $19 \%$ ). In summary, a typical normal terminal seems to grow by the slow elongation of most of its parts, with little retraction. In contrast, segments of regenerated terminals show much larger elongations that either outweigh (singly innervated) or match (doubly innervated) the frequent retractions.

In addition to the parts of terminals that persisted between 7 and 13 weeks, many segments appeared de novo or disappeared entirely over this interval. Figure $9 C$ shows that a substantial number of new branches was added in singly innervated junctions, averaging $40 \%$ of the number of segments present at 7 weeks. In contrast, the incidence of new branches was only $13 \%$ at doubly innervated junctions, a significant difference $(p<0.05)$. This difference was consistent with differences in the overall growth of terminals at singly and doubly innervated junctions, i.e., terminals at singly innervated junctions grew more. It was surprising that the two groups of terminals did not differ in the percentage of branch segments that disappeared. On average, $\sim 18 \%$ of the segments present at 7 weeks were deleted by 13 weeks in both singly and doubly innervated junctions. New branches and branch deletions were relatively rare in normal intact terminals (Herrera et al., 1990). The finding that singly and doubly reinnervated junctions suffer retraction and disappearance of terminal branches to about the same extent reinforces the conclusion that double innervation principally affects the tendency for terminal growth.

\section{Intramuscular gradients in the precision of reinnervation}

We observed that the precision of reinnervation varied with the position of junctions within the muscle. This positional effect was most striking when junctions at distal locations, far from the point where the sartorius nerve entered the muscle, were compared with proximal junctions, defined as those close to the point of nerve entry. Reinnervation at distal junctions seemed more faith- 

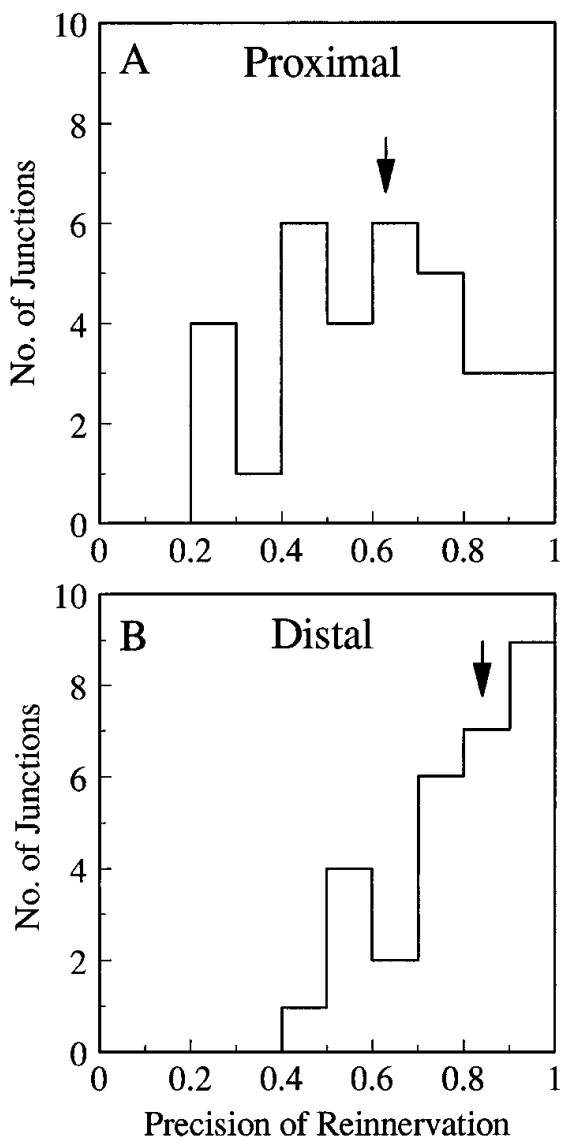

Figure 10. Within the sartorius muscle, distal junctions regenerated more precisely and completely than proximal junctions. Graphs show frequency distributions of an index for precision of reinnervation. $A$ shows data for 32 proximal junctions, and $B$ shows data for 29 distal junctions. Arrows indicate median values of 0.63 for proximal junctions and 0.84 for distal junctions. Distributions are significantly different $(p<0.01)$.

ful to the original pattern of the synapse. To examine this further, we selected 29 distal junctions and 32 proximal junctions, based solely on distance from the point of nerve entry. On average, the regenerated size of distal junctions was more than twice that of proximal junctions (distal $448 \pm 44 \mu \mathrm{m}$; proximal $196 \pm 12 \mu \mathrm{m}$; mean $\pm \mathrm{SEM}$ ). The precision of reinnervation was measured as in Figure $6 A$, and results are presented in Figure 10. There was a significant difference between proximal (Fig. 10 $A$ ) and distal junctions (Fig. 10B), with the distal results skewed toward the higher values. Median precision was only 0.63 for proximal junctions [interquartile range (i.q.r.) $0.45-0.77$ ) but 0.84 for distal junctions (i.q.r. 0.72-0.92), a significant difference $(p<0.01)$. Viewed another way, more than half $(54 \%)$ of the distal junctions showed precision greater than 0.8 , whereas only about one fifth (19\%) of proximal junctions regenerated that precisely.

\section{DISCUSSION}

\section{Synapse elimination and competition in reinnervated frog neuromuscular junctions}

The sartorius of Rana pipiens is typical among vertebrate muscles in that reinnervation results in high levels of polyneuronal innervation, most of which is subsequently eliminated. Specifically, double innervation of neuromuscular junctions falls from $\sim 50 \%$ shortly after reinnervation to $\sim 20 \%$ over the next 12 weeks after nerve crush (Herrera and Werle, 1990). Among the reinnervated junctions we observed in vivo, some were found to be doubly innervated at 13 weeks. Between 7 and 13 weeks after injury, we saw little evidence for synapse elimination, which we define as the total elimination of one or more axonal inputs from a junction. We did not observe wholesale retraction or growth of one input compared with another. Doubly innervated junctions displayed regressive changes, however, and did so more frequently than singly innervated junctions. These findings are consistent with the view that there is ongoing competition between terminals at doubly innervated junctions, even in the absence of synapse elimination. Our results also are consistent with earlier findings that junctions on dually innervated fibers in frogs are substantially weaker and smaller than junctions on singly innervated fibers, both in intact muscles (Haimann et al., 1981a; Nudell and Grinnell, 1983) and reinnervated muscles (Grinnell et al., 1979; Haimann et al., 1981b).

Clearly, these interactions are competitive, inasmuch as the behavior of one input is altered when a second input is present (Colman and Lichtman, 1993). If synapse elimination were complete in the first few weeks after nerve crush, then the cases of double innervation we observed at 7 weeks would be the surviving cases, those which were not eliminated, perhaps because the ability of the inputs to compete was balanced. In previous studies of doubly reinnervated junctions that survived elimination, we found that the two synapses tended to be balanced in synaptic efficacy (Werle and Herrera, 1988). This balance, and the double innervation, can persist for up to 2 years (Werle and Herrera, 1991). The present study suggests that the ability of the synapses to grow and retract also may be balanced.

\section{Dynamic remodeling of regenerated motor nerve terminals}

Regenerated motor nerve terminals in the present study were more dynamic than normal terminals (Herrera et al., 1990). Simultaneous growth and retraction frequently occurred in different parts of the arborization of single terminals. The various parts of nerve terminals may act independently, perhaps responding to highly localized stimuli. Alternatively, growth and retraction may be related if, for example, recycling of materials from retracted branches facilitates growth in other branches. Regardless of these relationships, the state of the nerve terminal is well represented as an equilibrium between opposing processes. Shifts in the balance cause net growth (as in singly reinnervated junctions), no change (as in doubly reinnervated junctions), or retraction.

Why, in general, should regenerated terminals be more dynamic than intact terminals? The answer probably involves the mechanisms proposed to regulate the structure of adult neuromuscular junctions. For example, remodeling may result from changes in Schwann cell processes, which in rats seem to be important in sprouting of motor axons and terminals and in guiding regeneration (Reynolds and Woolf, 1992; Son and Thompson, 1995a,b). Although we did not visualize Schwann cell processes, we frequently saw movement of Schwann cell somata in frog junctions, both after reinnervation (Figs. 3, 4) and in normal conditions (Fig. 4 in Herrera et al., 1990) (see also Chen et al., 1991). Alternatively, remodeling may result from changes in neurotrophic regulation after reinnervation. It has been proposed that muscle-derived neurotrophin-4 is responsible for presynaptic growth and remodeling (Funakoshi et al., 1995). Finally, it has been suggested that plasticity results from an imbalance between the activation of extracellular proteases by the muscle fiber and activity-dependent release of protease inhibitors (Liu et al., 1994). 
It is interesting to note that in each of these mechanisms, activity seems to play a key role (Colman and Lichtman, 1993). The higher levels of polyneuronal innervation that follow reinnervation may result in greater asynchrony between pre- and postsynaptic activity, which could trigger plasticity according to Hebbian rules (Hebb, 1949; Balice-Gordon and Lichtman, 1994). Whatever the mechanism, differences in the regulation of nerve terminal structure at singly and doubly innervated junctions favor growth in the former and regression in the latter.

\section{Precision of reinnervation of former synaptic sites}

The prevailing hypothesis is that reinnervation of neuromuscular junctions is highly precise. This view was established in histological studies of the frog cutaneous pectoris muscle (Letinsky et al., 1976; DeCino, 1981; Ding, 1982) and was confirmed by repeated observation of the mouse sternomastoid (Rich and Lichtman, 1989). Nevertheless, our previous histological observations of frog sartorius junctions indicated that reinnervation may be less precise than generally appreciated (Werle and Herrera, 1988, 1991). We therefore reexamined this question using repeated in vivo observations. When viewed 7 weeks after injury, many branches of regenerated sartorius terminals were indeed located in gutters formerly occupied by branches of the intact terminal. However, terminals behaved in two ways that greatly reduced the overall precision of reinnervation. First, many former gutters were not occupied at 7 weeks and remained vacant even at 10 and 13 weeks, suggesting that they were abandoned either in the sense of never being reinnervated or being reinnervated only transiently. Second, nerve terminal branches formed new contacts on patches of sarcolemma that were not previously seen to bear synapses. It is possible that some of these new sites previously were innervated, but terminals retracted before the first observation. However, terminal retraction is relatively infrequent in normal junctions, as evidenced both by histology (Wernig et al., 1980) and repeated observation (Herrera et al., 1990). We conclude, therefore, that most of these new sites were innervated de novo. Thus, although former synaptic gutters are clearly attractive to regenerating terminals, they also can be ignored in favor of nearby sarcolemma that has never been innervated.

It is possible that imprecise reoccupation of former synaptic gutters can be explained by some deficit in the mechanisms that specify or stabilize sites of reinnervation. For example, Schwann cells in denervated sartorius nerve sheaths could be relatively ineffective in supporting or directing the regeneration of motor axons. Terminal Schwann cells at denervated junctions may not extend processes to guide regenerating axons as in the rat (Son and Thompson, 1995a). At the denervated junctions, molecules in the extracellular matrix or postsynaptic membrane that specify sites of reinnervation or are necessary for synaptic maintenance may be less stable. AChRs, for example, are known to disappear from synaptic gutters that are abandoned when terminals retract spontaneously (Krause and Wernig, 1985) or when junctions are only partially reinnervated (Grinnell and Do, 1991). Repeated in vivo observations of frog junctions showed that agrin disappears along with AChR as quickly as $7 \mathrm{~d}$ after a gutter is abandoned (Werle and Sojka, 1996). In adult mice, the loss of AChRs from parts of the junction renders such sites unattractive to innervation (Rich and Lichtman, 1989; Balice-Gordon and Lichtman, 1994). Long-term denervated frog junctions also show a progressive decline in agrin (Reist et al., 1987) and cholinesterase (Wernig et al., 1980; Herrera et al., 1990).

The present results may be integrated with the explanations listed above by hypothesizing that the difference between precise reinnervation in the cutaneous pectoris or sternomastoid and imprecise reinnervation in the sartorius relates to the number of neuromuscular junctions per fiber. In the cutaneous pectoris and sternomastoid, fibers are innervated at a single junction. In frogs of the size we used, sartorius fibers bear two or three junctions (Banner and Herrera, 1986). Most of these junctions are capable of generating action potentials that conduct along the full length of the fiber. Assuming that all junctions are not reinnervated simultaneously, axons arriving at the second or third junctions will encounter fibers that already have been activated by the first junction reinnervated. The link between multisite innervation and the imprecision of reinnervation may lie in the activity dependence of mechanisms that specify sites of reinnervation. It is known, for example, that functional blockade of AChRs only triggers nerve terminal retraction when the muscle fiber is active, not when it is inactive (Balice-Gordon and Lichtman, 1994). Likewise, processes extended by denervated Schwann cells that may guide regenerating axons retract once activity is restored (Son and Thompson, 1995a). By restoring activity before reinnervation is complete, multisite innervation could cause AChRs and Schwann cell processes to be reduced at junctions that still are denervated, leading to a reduction in the precision and completeness of reinnervation at such junctions.

The present methods did not allow visualization of terminals in the first few weeks of reinnervation. It remains possible, therefore, that the initial reoccupation of former gutters is precise. If so, then these initial contacts must be unstable, because when we began observations a few weeks later, they had retracted. The difference between reinnervation that is imprecise from the outset and reinnervation that is precise early then imprecise later, may simply be in the timing of the changes that destabilize contacts. The more rapid these changes, the more the system would be characterized as initially imprecise. The slower the changes are to develop, the more the system would seem initially precise, with imprecision developing later. The rapidity of the destabilizing changes may in turn depend on their sensitivity to altered activity. Experiments to test these alternatives are in progress.

These considerations do not explain the surprising finding that the precision of reinnervation varied with position in the muscle. Junctions that were most distant from the point where the sartorius nerve entered seemed to form a separate group in which regeneration more faithfully reproduced the pattern of the former terminal. Incompletely reinnervated junctions, including some that only vaguely resembled the original terminal, were found throughout the remainder of the muscle. It could be that there is some topographic specification in the motor innervation of the sartorius, such that reinnervation by a particular portion of the motoneuron pool is more favored in the distal part of the muscle.

Although the frog neuromuscular junction is dynamic under normal conditions, the present results show that it becomes even more so after nerve injury. The outcome of regeneration and reinnervation is not a return to normality, however. Neuromuscular connections remain plastic for a considerable period, perhaps permanently. It is hoped that additional study of such plasticity at this well-characterized synapse will advance our understanding of the cellular and molecular mechanisms of synaptic competition and stabilization and the sequelae of peripheral nerve injury. 


\section{REFERENCES}

Astrow SH, Pitaevski V, Dorlöchter M, Herrera AA (1993) Physiology and morphology of reinnervated frog neuromuscular junctions observed in vivo with RH dye. Soc Neurosci Abstr 19:645.

Balice-Gordon RJ, Lichtman JW (1990) In vivo visualization of the growth of pre- and postsynaptic elements of mouse neuromuscular junctions. J Neurosci 10:894-908.

Balice-Gordon RJ, Lichtman JW (1994) Long-term synapse loss induced by focal blockade of postsynaptic receptors. Nature 372:519-524.

Banner LR, Herrera AA (1986) Differences in synaptic efficacy at neuromuscular junctions in frog twitch muscles. J Physiol (Lond) 379:205-215.

Barry JA, Ribchester RR (1995) Persistent polyneuronal innervation in partially denervated rat muscle after reinnervation and recovery from prolonged nerve conduction block. J Neurosci 15:6327-6339.

Betz WJ, Mao F, Bewick GS (1992) Activity-dependent fluorescent staining and destaining of living vertebrate motor nerve terminals. J Neurosci 12:363-375.

Betz WJ, Ridge RM, Bewick GS (1993) Comparison of FM1-43 staining patterns and electrophysiological measures of transmitter release at the frog neuromuscular junction. J Physiol (Paris) 87:193-202.

Chen L, Folsom DB, Ko C-P (1991) The remodeling of synaptic extracellular matrix and its dynamic relationship with nerve terminals at living frog neuromuscular junctions. J Neurosci 11:2920-2930.

Colman H, Lichtman JW (1993) Interactions between nerve and muscle: Synapse elimination at the developing neuromuscular junction. Dev Biol 156:1-10.

DeCino P (1981) Transmitter release properties along regenerated processes at the frog neuromuscular junction. J Neurosci 1:308-317.

Ding R (1982) Lack of correlation between physiological and morphological features of regenerating frog neuromuscular junctions. Brain Res 253:47-55.

Dunia R, Herrera AA (1993) Synapse formation and elimination during growth of the pectoral muscle of Xenopus laevis. J Physiol (Lond) 469:501-509.

Funakoshi H, Belluardo N, Arenas E, Yamamoto Y, Casabona A, Persson H, Ibanez CF (1995) Muscle-derived neurotrophin-4 as an activitydependent trophic signal for adult motor neurons. Science 269:1495-1499.

Grinnell AD, Do J (1991) Effect of denervation and regeneration on the stability of junctional ACh receptors in mature frog muscles. In: Plasticity of motoneuronal connections (Wernig A, ed), pp 151-161. Amsterdam: Elsevier.

Grinnell AD, Herrera AA (1980) Physiological regulation of synaptic effectiveness at frog neuromuscular junctions. J Physiol (Lond) 307:301-317.

Grinnell AD, Letinsky MS, Rheuben MB (1979) Competitive interaction between foreign nerves innervating frog skeletal muscle. J Physiol (Lond) 289:241-262.

Haimann C, Mallart A, Tomás I Ferré J, Zilber-Gachelin NF (1981a) Patterns of motor innervation in the pectoral muscle of Xenopus laevis: evidence for possible synaptic remodelling. J Physiol (Lond) 310:241-256.

Haimann C, Mallart A, Tomás I Ferré J, Zilber-Gachelin NF (1981b) Interaction between motor axons from two different nerves reinnervating the pectoral muscle of Xenopus laevis. J Physiol (Lond) 310:257-272.

Hebb DO (1949) The organization of behavior. New York: Wiley.

Herrera AA (1984) Polyneuronal innervation and quantal transmitter release in formamide-treated frog sartorius muscles. J Physiol (Lond) 355:267-280

Herrera AA, Banner LR (1990) The use and effects of vital fluorescent dyes: observation of motor nerve terminals and satellite cells in living frog muscles. J Neurocytol 19:67-83.

Herrera AA, Werle MJ (1990) Mechanisms of elimination, remodelling, and competition at frog neuromuscular junctions. J Neurobiol 21:73-98.
Herrera AA, Grinnell AD, Wolowske B (1985) Ultrastructural correlates of experimentally altered transmitter release efficacy in frog motor nerve terminals. Neuroscience 16:491-500.

Herrera AA, Banner LR, Nagaya N (1990) Repeated, in vivo observation of frog neuromuscular junctions: remodelling involves concurrent growth and retraction. J Neurocytol 19:85-99.

Herrera AA, Banner LR, Werle MJ, Regnier M, Nagaya N (1991) Postmetamorphic development of neuromuscular junctions and muscle fibers in the frog cutaneous pectoris. J Neurobiol 22:15-28.

Hill RR, Robbins N (1991) Mode of enlargement of young mouse neuromuscular junctions observed repeatedly in vivo with visualization of pre- and postsynaptic borders. J Neurocytol 20:183-194.

Ko C-P (1981) Electrophysiological and freeze fracture studies of changes following denervation at frog neuromuscular junctions. J Physiol (Lond) 321:627-639.

Krause M, Wernig A (1985) The distribution of acetylcholine receptors in the normal and denervated neuromuscular junction of the frog. J Neurocytol 14:765-780.

Langenfeld-Oster B, Dorlöchter M, Wernig A (1993) Regular and photodamage-induced remodelling in vitally stained frog and mouse neuromuscular junctions. J Neurocytol 22:517-530.

Letinsky MS, DeCino P (1980) Histological staining of pre- and postsynaptic components of amphibian neuromuscular junctions. J Neurocytol 9:305-320.

Letinsky MS, Fischbeck KH, McMahan UJ (1976) Precision of reinnervation of original synaptic sites in frog muscle after a nerve crush. J Neurocytol 5:691-718.

Lichtman JW, Magrassi L, Purves D (1987) Visualization of neuromuscular junctions over periods of several months in living mice. J Neurosci 7:1215-1222.

Liu Y, Fields RD, Fitzgerald S, Festoff BW, Nelson PG (1994) Proteolytic activity, synapse elimination, and the Hebb synapse. J Neurobiol 25:325-335.

McArdle JJ (1975) Complex end-plate potentials at the regenerating neuromuscular junction of the rat. Exp Neurol 49:29-63.

Nudell BM, Grinnell AD (1983) Regulation of synaptic position, size, and strength in anuran skeletal muscle. J Neurosci 3:161-176.

Reist NE, Magill C, McMahan UJ (1987) Agrin-like molecules at synaptic sites in normal, denervated, and damaged skeletal muscles. J Cell Biol 105:2457-2469.

Reynolds ML, Woolf CJ (1992) Terminal Schwann cells elaborate extensive processes following denervation of the motor endplate. J Neurocytol 21:50-66.

Rich MM, Lichtman JW (1989) In vivo visualization of pre- and postsynaptic changes during synapse elimination in reinnervated mouse muscles. J Neurosci 9:1781-1805.

Son YJ, Thompson WJ (1995a) Schwann cell processes guide regeneration of peripheral axons. Neuron 14:125-132.

Son YJ, Thompson WJ (1995b) Nerve sprouting in muscle is induced and guided by processes extended by Schwann cells. Neuron 14:133-141.

Werle MJ, Herrera AA (1988) Synaptic competition and the elimination of polyneuronal innervation following reinnervation of adult frog sartorius muscles. J Neurobiol 19:465-481.

Werle MJ, Herrera AA (1991) Elevated levels of polyneuronal innervation persist for as long as two years in reinnervated frog neuromuscular junctions. J Neurobiol 22:97-103.

Werle MJ, Sojka AM (1996) Anti-agrin staining is absent at abandoned synaptic sites of frog neuromuscular junctions. J Neurobiol 30:293-302.

Wernig A, Herrera AA (1986) Sprouting and remodeling at the nervemuscle junction. Prog Neurobiol 27:251-291.

Wernig A, Pecot-Dechavassine M, Stover H (1980) Sprouting and regression of the nerve at frog neuromuscular junction in normal conditions and after prolonged paralysis with curare. J Neurocytol 9:277-303.

Wigston DJ (1989) Remodeling of neuromuscular junctions in adult mouse soleus. J Neurosci 9:639-647. 